\title{
Perfil de crianças e adolescentes internados em uma unidade de tratamento de queimados
}

\author{
Profile of children and adolescents hospitalized in a burn treatment unit
}

Perfil de niños y adolescentes hospitalizados en una unidad de tratamiento de quemaduras

Dienia Andrade dos Santos ${ }^{1}$, Isabela Mie Takeshita², Claudirene Milagres Araújo², Mara Rúbia Maciel Cardoso do Prado ${ }^{3}$, Camila Mendes dos Passos ${ }^{3}$, Bruno David Henriques ${ }^{3}$, Lílian Fernandes Arial Ayres ${ }^{3}$, Thiago Filipe Novato ${ }^{4}$, Fernanda de Souza ${ }^{4}$, Luciana Ramos de Moura ${ }^{3 *}$.

\section{RESUMO}

Objetivo: Analisar o perfil clínico-epidemiológico de crianças e adolescentes, vítimas de queimaduras, atendidas em um hospital de referência no estado de Minas Gerais. Métodos: Estudo epidemiológico, descritivo, retrospectivo-documental e quantitativo. Amostra de 251 prontuários de crianças e jovens com 0 19 anos internados em um centro de tratamento de queimados e UTI pediátrica. Resultados: Observou-se maior predominância de internações em indivíduos do sexo masculino (59\%); a faixa etária mais acometida foi entre $1-2$ anos (27\%). A escaldadura foi o agente causal mais frequente $(46 \%)$; o tipo de queimadura mais prevalente foi a de segundo grau profundo $(54 \%)$; os membros superiores $(63 \%)$ foram os mais acometidos e a média de tempo de internação foi 29,62 dias. Conclusão: Visto que o perfil clínico-epidemiológico de crianças e adolescentes, vítimas de queimaduras, apresentaram uma variação relacionado aos agentes causais de acordo com a faixa etária e a maioria desses acidentes ocorreu na própria residência, é necessário investir em estratégias intersetoriais de sensibilização para prevenir queimaduras, onde medidas preventivas simples podem ser eficazes.

Palavras-chave: Queimaduras, Unidades de queimados, Criança.

\begin{abstract}
Objective: To analyze the clinical-epidemiological profile of children and adolescents, victims of burns, attended at a reference hospital in the state of Minas Gerais. Methods: Epidemiological, descriptive, retrospective-documentary and quantitative study. Sample of 251 medical records of children and young people aged 0-19 years admitted to a burn treatment center and pediatric ICU. Results: There was a greater predominance of admissions in males (59\%); the most affected age group was between 1-2 years (27\%). Scald was the most frequent causal agent $(46 \%)$; the most prevalent type of burn was deep second degree (54\%); the upper limbs (63\%) were the most affected and the average length of stay was 29.62 days. Conclusion: Since the clinical and epidemiological profile of children and adolescents, victims of burns, presented a variation related to the causal agents according to age group and the majority of these accidents occurred at home, it is necessary to invest in intersectoral awareness-raising strategies for prevent burns, where simple preventative measures can be effective.
\end{abstract}

Keywords: Burns, Burned units, Kid.

${ }^{1}$ Fundação Hospitalar do Estado de Minas Gerais, Belo Horizonte - MG.

2 Faculdade Ciências Médicas de Minas Gerais, Belo Horizonte - MG.

3 Universidade Federal de Viçosa, Viçosa - MG. *E-mail: lucianamoura2903@gmail.com

${ }^{4}$ Centro Universitário de Belo Horizonte (UniBH), Belo Horizonte - MG. 


\section{RESUMEN}

Objective: Analizar el perfil clínico-epidemiológico de niños y adolescentes víctimas de quemaduras atendidos en un hospital de referencia en el estado de Minas Gerais. Métodos: Estudio epidemiológico, descriptivo, retrospectivo-documental y cuantitativo. Muestra de 251 historias clínicas de niños y jóvenes de 0 a 19 años ingresados en un centro de tratamiento de quemaduras y $\mathrm{UCl}$ pediátrica. Resultados: hubo mayor predominio de ingresos en el sexo masculino (59\%); el grupo de edad más afectado fue el de 1-2 años $(27 \%)$. Las escaldaduras fueron el agente causal más frecuente $(46 \%)$; el tipo de quemadura más prevalente fue de segundo grado profundo (54\%); los miembros superiores $(63 \%)$ fueron los más afectados y la estancia media fue de 29,62 días. Conclusión: Dado que el perfil clínico y epidemiológico de los niños y adolescentes víctimas de quemaduras, presentó una variación relacionada con los agentes causales según el grupo de edad y la mayoría de estos accidentes ocurrieron en el hogar, es necesario invertir en estrategias intersectoriales de sensibilización. para prevenir quemaduras, donde las medidas preventivas simples pueden ser efectivas.

Palabras clave: Quemaduras, Unidades de quemados, Niño.

\section{INTRODUÇÃO}

A interação do infante com o meio é necessária para o desenvolvimento neuropsicomotor e consequente aquisição de habilidades cada vez mais complexas. Por outro lado, a vivência de novas experiências e curiosidades aumenta a vulnerabilidade de crianças e adolescentes aos acidentes, em especial os domésticos, como quedas, intoxicações, afogamentos e queimaduras. Esta última é considerada grave pela seriedade clínica e possibilidade de sequelas físicas, algumas irreversíveis (ANDRETTA IB, et al., 2013).

Queimaduras são lesões resultantes da ação do calor direto ou indireto sobre o corpo, ocorre a destruição da pele, são classificadas pelo nível de acometimento e extensão. A escaldadura, seguida do álcool, chama direta, choque elétrico e produtos químicos são seus principais agentes causadores. Promovem a morbidade infantil e provocam, além do comprometimento estético, danos metabólicos e distúrbios hidroeletrolíticos, levando à insuficiência renal e infecções graves, limitações, sequelas e tratamentos corretivos por toda infância e adolescência, constituindo um grande problema de saúde pública no Brasil (LUCENA EV e FIGUEIREDO TP, 2017).

Nos últimos cinco anos o Brasil registrou 171.551 internações relacionadas a queimaduras em menores de 19 anos. De acordo com o Ministério da Saúde entre 2013 e 2014 foram registrados mais de 15 mil casos de internações por queimaduras em crianças entre 0 e 10 anos (MINISTÉRIO DA SAÚDE, 2018).

Acidentes e violências provocam queimaduras, estas oneram gastos aos serviços de saúde, reduzem a produtividade, acarretam sequelas e precoces óbitos, além de impactos sociais e familiares. Sua prevenção e tratamento deve ser discutida em instâncias governamentais e acadêmicas, priorizando a articulação intersetorial (educação, saúde e segurança e sociedade civil) (MESCHIAL WC, et al., 2020). Ressalta-se que a maior incidência de queimaduras na infância ocorre em ambiente doméstico e poderiam ser evitadas com a adoção de medidas relativamente simples (BARCELLOS LG, et al., 2018).

O presente estudo objetivou analisar o perfil de crianças e adolescentes, vítimas de queimaduras, admitidos em uma Unidade de Tratamento de Queimados (UTQ) no estado de Minas Gerais.

\section{MÉTODOS}

Estudo epidemiológico, descritivo, retrospectivo-documental e de natureza quantitativa. A pesquisa foi realizada em uma Unidade de Tratamento de Queimados no estado de Minas Gerais.

Foram analisados, retrospectivamente, dados de prontuários de 251 crianças e adolescentes internados na UTQ e CTI pediátrico no período de janeiro a dezembro em 2017. Foram coletados registros de crianças e adolescentes no livro do Sistema de Arquivo Médicos e Estatísticos. Cada registro do período foi pesquisado no sistema eletrônico do hospital. Os critérios de inclusão foram: diagnóstico clínico de queimadura, acompanhamento na UTQ e CTI pediátrico por um período mínimo de 24 horas. 
O instrumento de coleta de dados foi elaborado a partir das principais variáveis envolvidas na ocorrência das queimaduras de acordo com a literatura científica. Foram avaliados sexo, local de residência, áreas acometidas, tempo de internação em faixa de dias, agentes causais de queimaduras como a escaldadura, chama direta, álcool, choque elétrico, e outros. Houveram considerações como as internações por zetaplastia, apesar de não se configurarem agente causal, foram incluídas por serem causa de reinternações por queimaduras, devido a sequelas graves e importantes (LAITANO FF, et al., 2013). Além disso, o mesmo paciente poderia apresentar diversas áreas queimadas, ultrapassando $100 \%$ na somatória dos resultados (SANTANA ME, et al., 2018).

Foram avaliados o grau de acometimento, onde excluíram prontuários referentes à reinternações por zetaplastia. Avaliou-se a extensão da queimadura por faixa de porcentagem da superfície corpórea acometida, neste caso, também foram excluídos prontuários de internações para zetaplastia (LAITANO FF, et al., 2013). Foram analisados os procedimentos realizados na área queimada, como debridamentos cirúrgicos, enxertias, zetaplastias, amputações, retalhos, realização de curativos, fasciotomias e escarotomias, esta variável pode ultrapassar $100 \%$ na somatória dos resultados, pois alguns pacientes passaram por mais de um procedimento (SANTANA ME, et al., 2018).

A zetaplastia é um procedimento de correção de sequelas, não sendo um agente etiológico, porém foi abordada nesse estudo, por ser uma das causas de reinternações frequentes, sendo responsável por 18 (7\%) das internações no período.

Os dados foram armazenados em planilha do programa Microsoft Exceße, para as associações entre as variáveis, o programa estatístico SPSS $\AA$. Os resultados foram apresentados através de estatística descritiva, percentuais, valores absolutos, médias e medianas. As associações entre as variáveis foram analisadas pelo teste de Kruskal-Wallis. Esses resultados foram considerados significativos para uma probabilidade de significância inferior a $5 \%(p<0,05)$.

Esta pesquisa respeitou os preceitos éticos da legislação vigente e foi aprovada no Comitê de Ética em Pesquisa sob CAAE: 87010318.3.0000.5093.

\section{RESULTADOS}

Foram analisados 251 prontuários. Crianças e adolescentes do sexo masculino estiveram mais frequentemente envolvidas em acidentes que cursaram com queimaduras, foram 147 casos (59\%), por outro lado, 104 (41\%) para o sexo feminino.

A faixa etária mais afetada por acidentes envolvendo queimaduras foi a de 1-2 anos de idade, com $68(27 \%)$. Em segundo lugar, os adolescentes de $16-19$ anos com $57(23 \%)$. As outras faixas etárias apresentaram percentuais menores: 8-12 anos (13\%), 5-7 anos (12\%), 13-15 anos (11\%), 3-4 anos (10\%), 711 anos (3\%) e 0-28 dias, 29dias-3meses e 4-6meses (0,4\%).

Houve maior proporção de crianças e adolescentes hospitalizados por queimaduras provenientes do interior de Minas Gerais, 139(55\%) casos. A região metropolitana de Belo Horizonte respondeu por 65(26\%) e a residente na capital mineira por $47(19 \%)$.

A escaldadura foi o principal agente causador da injúria térmica, acometendo 116(46\%) vítimas, seguida da chama direta, $40(16 \%)$. O álcool foi descrito como agente causal em $37(15 \%)$. Já o choque elétrico foi responsável por $25(10 \%)$ e, 15(6\%), foram provocados por outros agentes. A zetaplastia foi responsável por 18(7\%).

O Gráfico abaixo apresenta a relação entre o agente causal e a faixa etária acometida pela queimadura. As crianças menores de 4 anos são as mais acometidas por escaldadura, 87(46\%), com maior destaque para as crianças entre 1 a 2 anos de idade 62(24,5\%). Já indivíduos entre 13 e 15 anos foram mais vitimizados pelo choque elétrico, 10(37\%). O álcool, por sua vez, foi o agente causal mais frequente entre adolescentes de 16 a 19 anos, 24(42\%) (Gráfico 1). 
Gráfico 1 - Associação entre a faixa etária e agente causal entre crianças e adolescentes, vítimas de queimaduras, internados em UTQ.

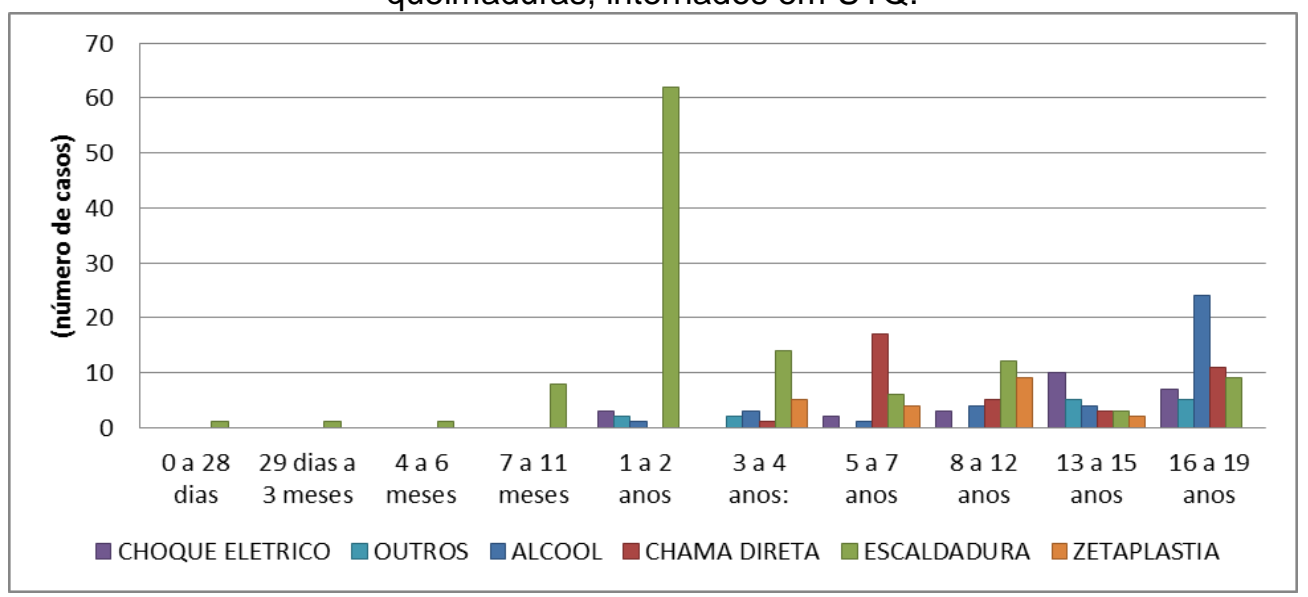

Fonte: Moura LR, et al., 2021.

Com relação a área corpórea afetada, os membros superiores (MMSS) foram os mais afetados, com ocorrência de 158(63\%), seguido do tórax com 157(62\%). A região da cabeça foi descrita em 117(46\%). Os membros inferiores (MMII) foram acometidos em 102(40\%). O abdome foi relatado como área atingida em $78(31 \%)$, genitália e glúteos em $20(8 \%)$. Ressalta-se que alguns pacientes apresentaram mais de um local acometido, somando-se mais de $100 \%$.

Com relação ao grau da queimadura, as lesões de segundo grau superficial foram percebidas em 75(30\%); as de segundo grau profundo em 135(54\%); as de terceiro grau em 73(29\%) e a exposição óssea foi descrita em $13(5 \%)$.

O Gráfico a seguir apresenta a associação entre agente causal e grau da queimadura. Entre os pacientes acometidos por escaldadura, predominaram as lesões de segundo grau superficial com 46(62,2\%) e de segundo grau profundo com $57(68,7 \%)$. Nos pacientes vítimas de queimadura de terceiro grau o agente mais frequente foi a chama direta, descrita em 7(41\%). No grupo de pacientes com queimaduras de segundo e terceiro graus os agentes mais frequentes foram o álcool e a chama direta com 12(28,6\%) casos cada. Com exposição óssea, 10(76,9\%) foram vítimas de choque elétrico (Gráfico 2).

Gráfico 2 - Associação entre o grau da queimadura e agente causador entre crianças e adolescentes admitidos numa unidade de tratamento de queimados.

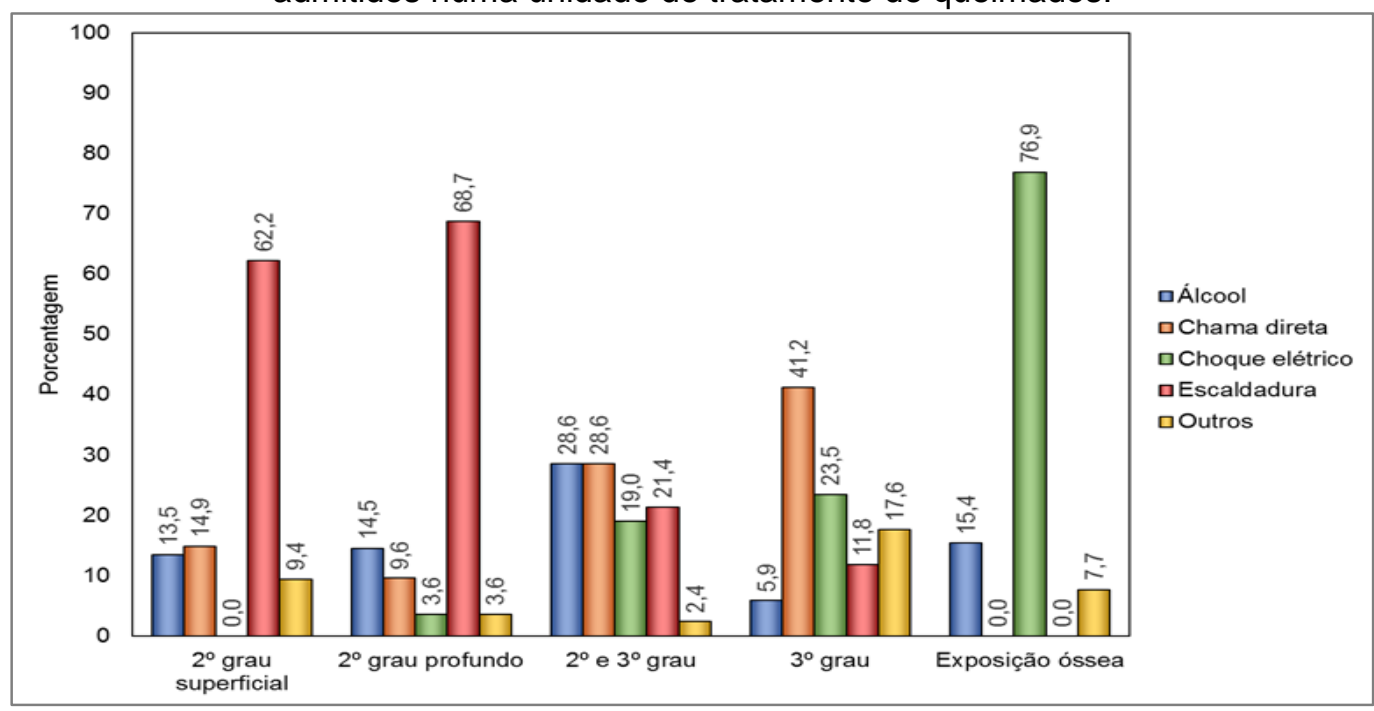

Fonte: Moura LR, et al., 2021.

A associação entre o grau da queimadura e mediana de idade evidenciou diferenças significativas entre os grupos $(p<0,05)$, sendo a queimadura com exposição óssea a mais frequente entre os indivíduos de idade 
mais avançada. Queimaduras de $2^{\circ}$ e de $3^{\circ}$ graus, por sua vez, foram mais frequentes na faixa etária dos 10 anos conforme exposto abaixo (Gráfico 3).

Gráfico 3 - Estratificação da relação entre o grau da queimadura e a mediana de idade entre crianças e adolescentes admitidos em UTQ.

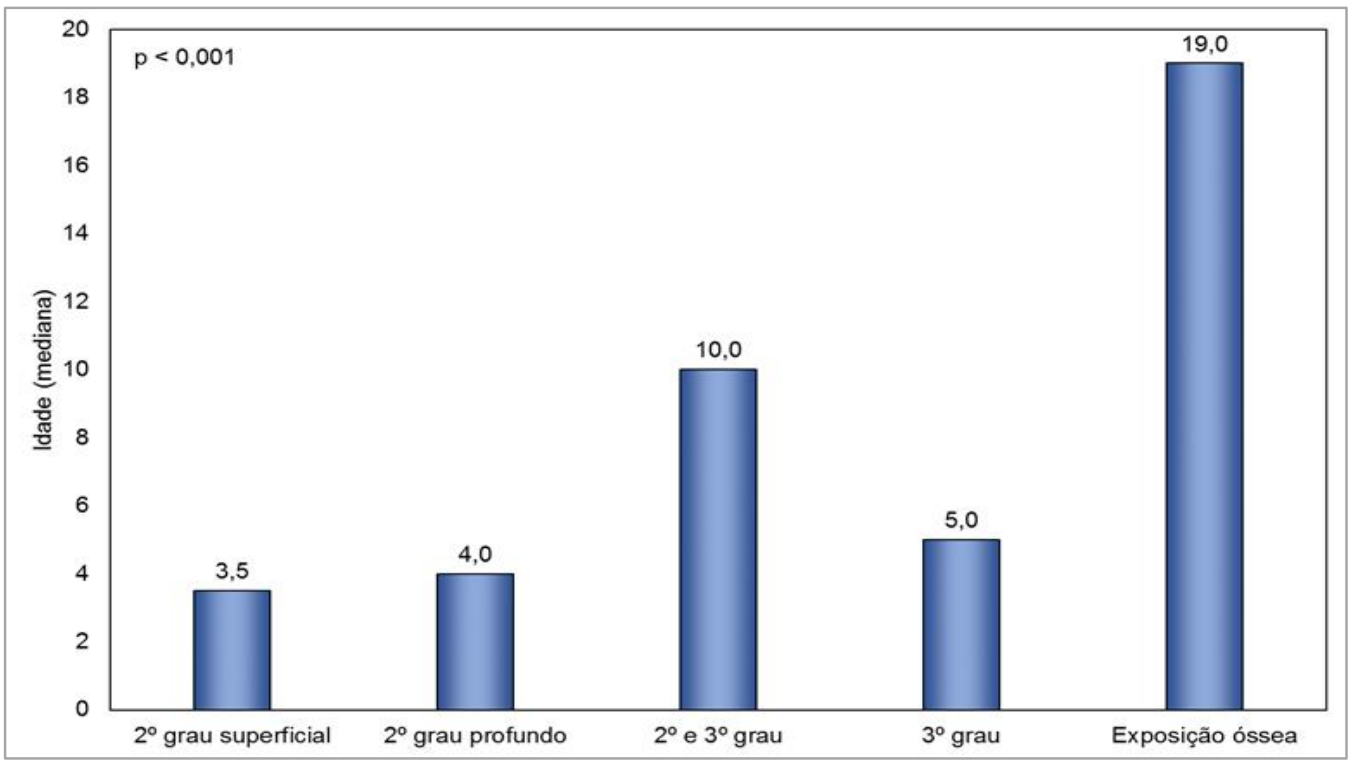

Fonte: Moura LR, et al., 2021.

Destaca-se que $114(49 \%)$ crianças e adolescentes apresentaram entre $11-20 \%$ da SCQ, seguidos por $80(35 \%)$ que apresentaram queimaduras em $1-10 \%$ do corpo. Outros $21(9 \%)$ foram vítimas de queimadura em $21-30 \%$ da superfície corporal; $10(4 \%)$ apresentaram $31-50 \%$ do corpo queimado e $8(3 \%)$ entre $51-80 \%$ da SCQ. Pacientes com mais de $81 \%$ da SCQ não foram descritos entre os internados. Foram excluídas 18 internações por zetaplastia, pois não se aplica a extensão de superfície corpórea acometida.

Com relação aos procedimentos realizados durante o período de internação hospitalar, 51(20\%) necessitaram apenas de curativos para o tratamento das lesões corpóreas. $O$ debridamento cirúrgico, por sua vez, foi necessário em 180(72\%). Foram submetidos à enxertia cutânea 148(59\%) e 7(3\%) realizaram escarotomia e fasciotomia. Amputações foram registradas em $5(2 \%)$. Foram submetidos a retalhos $9(4 \%)$ e $18(7 \%)$ internaram para correção de sequelas de queimaduras pregressas (zetaplastia). Ressalta-se, nesse contexto, que alguns pacientes realizaram mais de um procedimento relacionado à lesão por queimadura, 0 que justifica a somatória dos eventos superior a $100 \%$.

A maior parte dos pacientes (39\%) necessitaram de 16-30 dias de internação, enquanto 19\% permaneceram internados durante 31-45 dias. A média de internação dos pacientes foi de 29,62 dias, conforme detalhado (Gráfico 4).

Gráfico 4 - Tempo de internação entre crianças e adolescentes admitidos em UTQ.

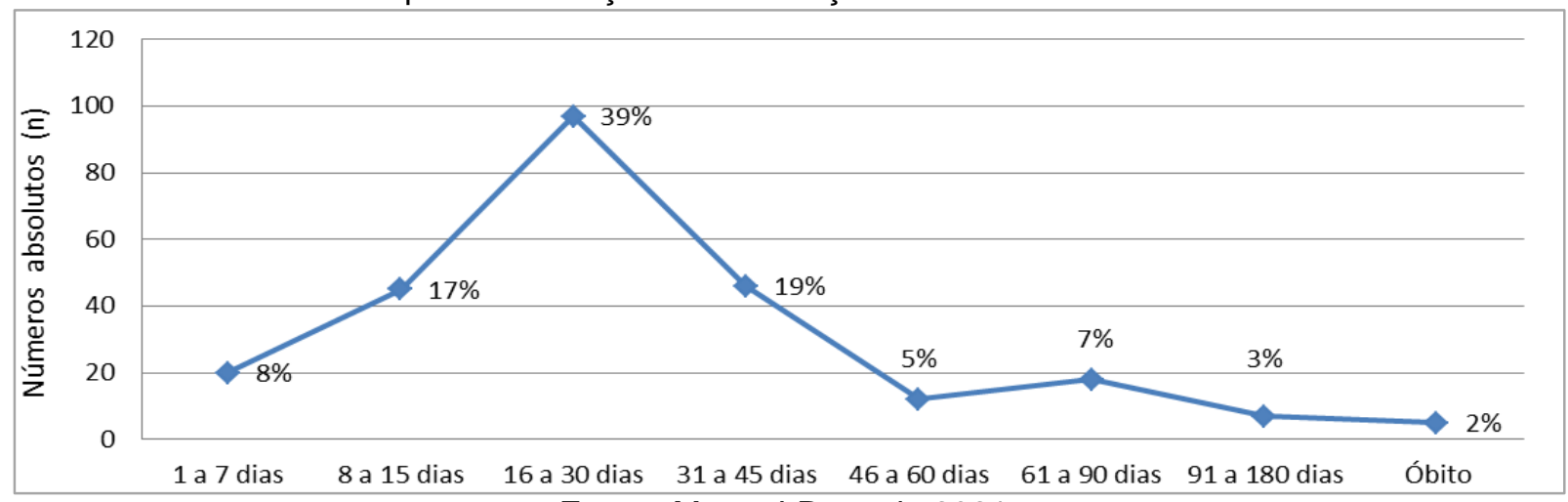

Fonte: Moura LR, et al., 2021. 
O Gráfico abaixo relaciona o tempo de internação ao grau da queimadura, foram verificadas diferenças significativas entre os grupos $(p<0,05)$. Casos com exposição óssea apresentaram maior tempo de internação, com mediana de 61 dias. Já os indivíduos com queimaduras de $2^{\circ}$ grau superficial e $3^{\circ}$ grau, apresentaram os menores tempos de internação com mediana de 16 e 20,5 dias, respectivamente (Gráfico 5).

Gráfico 5 - Associação entre o grau da queimadura e o tempo de internação entre crianças e adolescentes admitidos em UTQ.

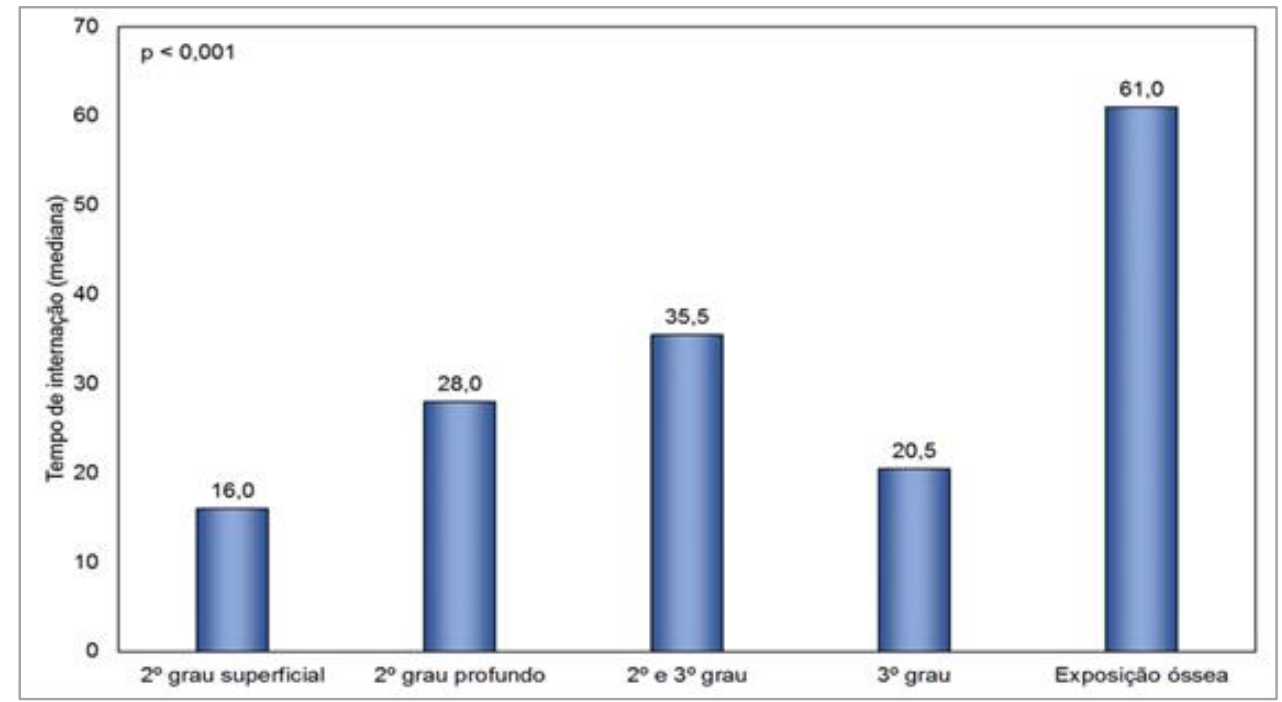

Fonte: Moura LR, et al., 2021.

A relação entre tempo de internação e o agente causal evidenciou que as internações que duraram de 61 a 90 dias ou mais, o choque elétrico foi o agente causal mais frequente. Já a escaldadura foi o agente mais prevalente nas internações de 8 a 45 dias de duração. Foram constatados 5 óbitos na UTI pediátrica, tendo como agente causal a chama direta, conforme evidenciado (Gráfico 6).

Gráfico 6 - Relação entre o agente causal da queimadura e o tempo de internação entre crianças e adolescentes atendidos em UTQ.

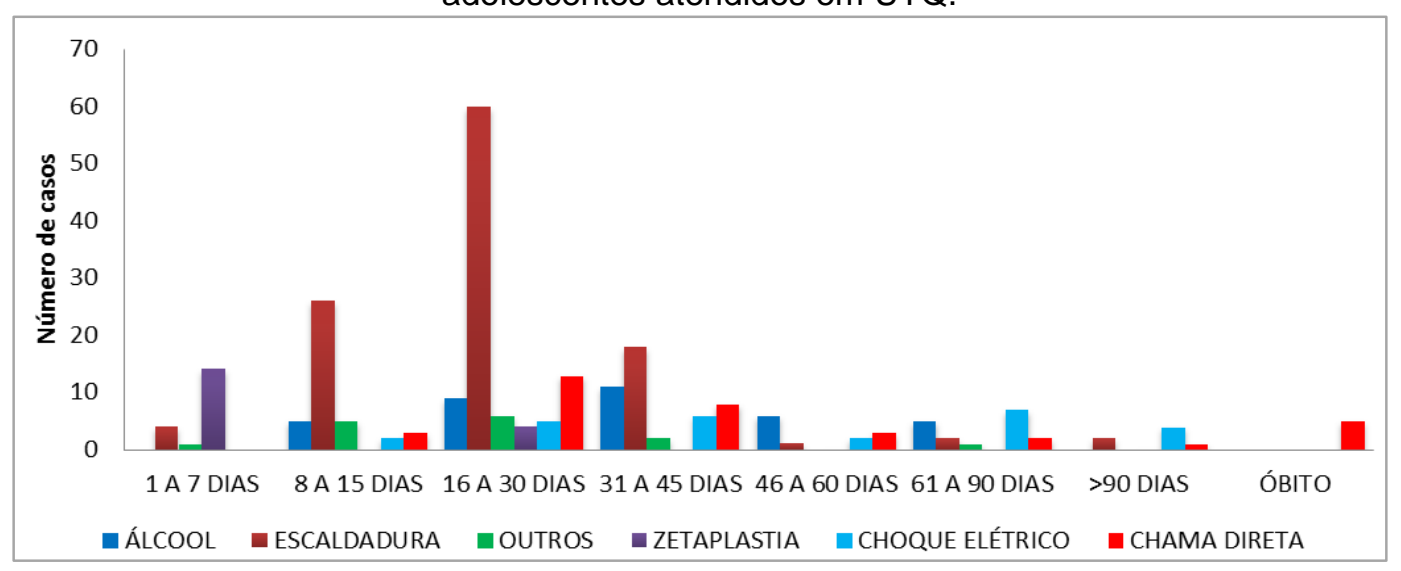

Fonte: Moura LR, et al., 2021.

\section{DISCUSSÃO}

No presente estudo, o sexo masculino foi responsável pelo maior número de queimaduras, com $59 \%$ dos casos e corrobora estudo na mesma UTQ, com maior frequência entre crianças e adolescentes do sexo masculino (LIMA JS, et al., 2016). Estudo realizado em São Paulo, entre 2012 e 2015, também destacou maior prevalência de queimaduras no sexo masculino (SANCHES PH, et al., 2017). Do mesmo modo, pesquisa realizada em Cuba por Hernandez CM, et al. (2018) evidenciou que, entre as crianças vítimas de 
queimaduras, há predomínio do sexo masculino. De acordo com esse mesmo autor, os meninos tendem a se envolver mais em brincadeiras de risco quando comparados às meninas e essas, por outro lado, recebem maior supervisão e proteção dos pais.

As crianças com idade entre 1-2 anos apresentaram maior número de internações por queimaduras e o agente causal mais prevalente foi a escaldadura, assim como identificado por Hernandez CM, et al. (2018), Sanches PH, et al. (2017) e Lima JS, et al. (2016). A escaldadura é causada por líquidos superaquecidos, como água para preparo de alimentos no ambiente domiciliar. Esta faixa etária é vulnerável a esse tipo de acidente em função do processo acelerado de desenvolvimento neuropsicomotor, curiosidade aguçada, menor coordenação motora, maior dependência de um adulto supervisor e, um instante de distração, pode repercutir em acidente (TAKINO MA, et al., 2016).

Estudo realizado no Canadá evidenciou que a imaturidade motora da criança associada a capacidade de lançar e puxar para si objetos próximos, como o prato de papinha ou a água da banheira, contribuem para a ocorrência de escaldaduras (ANDRETTA IB, et al., 2013). Esta realidade foi agravada durante a pandemia do SarsCov2, que impôs às famílias o isolamento domiciliar, medida importante para evitar a infecção, mas que, ao mesmo tempo, colocou desafios, pois crianças e adolescentes estão todo o tempo em casa, com pais sobrecarregados entre tarefas domésticas, home office e cuidados com os filhos sem rede de apoio (MARCHETI MA, et al., 2020). Houve aumento significativo da incidência de queimaduras relacionadas ao uso de álcool, devido uma maior disponibilidade do produto nas residências, utilizando-o para higienização das mãos, superfícies e objetos (BRITO AR, et al., 2021).

Entre 5-12 anos de vida, os acidentes causados por chama direta apresentaram maior frequência. Esse grupo de crianças e adolescentes apresenta relativa independência e pouco julgamento sobre as situações de risco: exploram novas brincadeiras e realizam experimentos com produtos que, muitas vezes, são inflamáveis. Já os acidentes com álcool estão entre os mais registrados entre adolescentes de 16-19 anos, a maioria deles ocasionados ao acender churrasqueiras, colocar fogo em lixo, ou situações de agressão (ANDRETTA IB, et al., 2013).

Adolescentes acima dos 13 anos foram mais vitimizados pelo choque elétrico. Essa ocorrência é frequente em brincadeiras que envolvem pipa que alcançam correntes elétricas de alta voltagem. Elas são mais graves devido à passagem da corrente pelo corpo que compromete músculos, órgãos, nervos, vasos sanguíneos e a libera enzimas musculares na corrente sanguínea, sobrecarrega rins e gera alterações metabólicas. $O$ tratamento das lesões por queimaduras elétricas é complexo, inclui debridamentos, enxertias, fasciotomias, retalhos locais e amputações (TONDINELI TH, et al., 2016). No Brasil, o nordeste sofreu com mais casos de mortes por choque elétrico (CRUZ BEZERRA AF, et al., 2020).

O interior de Minas Gerais foi responsável por mais internações por queimaduras, perfazendo $55 \%$ dos casos. Estudo realizado em 2012, no mesmo hospital, também apresentou maior registro de internações entre os pacientes procedentes das cidades interioranas (LIMA JS, et al., 2016). Em Recife e no Ceará evidenciaram maior frequência de queimaduras entre os residentes do interior e zona rural (LIRA PR, et al., 2017; JUNIOR EM, et al., 2014). A falta de centros especializados em queimaduras nessas localidades pode justificar a necessidade de transferência de crianças e adolescentes para realização do tratamento adequado nas capitais (SANTANA ME, et al., 2018).

As áreas corpóreas mais frequentemente acometidas na presente investigação foram membros superiores e tórax, dado que corrobora com os estudos de Silva IK, et al. (2015) e Santana ME, et al. (2018). A maior concentração de queimaduras nessas regiões do corpo relaciona-se à dinâmica da injúria térmica e posição em que a criança ou adolescente estavam no momento do acidente. Nesses casos é comum a vítima puxar, para si, o objeto ou o líquido aquecido, atingindo os braços. Fatalmente, o tórax também está mais exposto durante esse tipo de acidente (FRANCISCONI MH, et al., 2016).

No presente estudo as queimaduras de segundo grau profundo foram as mais incidentes, resultados semelhantes foram encontrados por Silva RL, et al. (2016), Silva IK, et al. (2015) e Leite VH, et al. (2017). Sobre a extensão da queimadura, 49\% apresentaram comprometimento de $11-20 \%$ da SCQ, seguido de $35 \%$ dos casos com 1-10\% da SCQ. Estudo realizado por Leão CE, et al. (2011), no mesmo hospital, aponta uma 
média de SCQ de 20,8\%. Há semelhança ainda com estudos de Santana ME, et al. (2018) e Barcellos LG, et al. (2018) onde médios queimados responderam pela maioria dos casos.

Os procedimentos mais prevalentes no período analisado foram os debridamentos e enxertias cutâneas. No mesmo local, Leão CE, et al. (2011), identificou elevada ocorrência de debridamentos e enxertias entre os queimados. A maioria das crianças necessitaram de, ao menos, um procedimento cirúrgico, sendo o debridamento seguido da enxertia, os mais frequentes (TAKINO MA, et al., 2016). Apesar do avanço promovido pelas coberturas e procedimentos que auxiliam e aceleram o tratamento e a cicatrização das queimaduras, o debridamento e a enxertia precoce ainda são considerados padrão ouro no tratamento por minimizarem os riscos de infecção (JUNIOR EM, et al., 2017).

No presente estudo, a média de internação foi de 29,62 dias (variando de 1-180 dias), valor maior que aquele encontrado no mesmo setor em dois estudos anteriores: Lima JS, et al. (2016) descreveu uma média de 22,36 dias (4-114 dias) e Leão CE, et al. (2011) uma média de 23,5 dias. Investigação conduzida em Londrina, apresentou média de 12 dias de internação em 2016 e de 16 dias em 2015 (TAKINO et al., 2016). O tempo de internação varia de acordo com a extensão da queimadura, agente causal, profundidade e fatores, como o estado de saúde da vítima. Internações, especialmente as prolongadas, representam grande trauma às crianças e adolescentes por acarretar intenso sofrimento físico associado a dor, além de maior risco de infecção hospitalar (LUCENA EV e FIGUEIREDO TP, 2017).

As vítimas de queimaduras por choque elétrico apresentaram maior tempo de internação (mais de 61 dias) e a maioria com queimaduras de 3 o grau e exposição óssea. Estudos de Filho RF, et al. (2014) e Arruda FC (2017) evidenciaram que quanto maior o grau de queimadura maior será o tempo de internação. Por outro lado, a chama direta, durante o período estudado, foi responsável por $5(2 \%)$ óbitos, porcentagem menor que aquela evidenciada no mesmo centro em estudo anterior no ano de 2012, onde havia 136 crianças e adolescentes internadas e a taxa de mortalidade foi de 2,9\% (LIMA JS, et al., 2016).

O tratamento especializado requer competência e conhecimentos acerca do trauma, avaliação da extensão e gravidade, além de atenção, por parte da equipe de saúde, às prováveis alterações fisiológicas que o corpo sofre a partir da exposição a cada tipo de agente causal. $O$ uso de terapêuticas apropriadas a cada evento minimiza o tempo de internação, as complicações e o sofrimento (SANTOS CA e SANTOS AA, 2017). Apesar dos esforços empregados nas UTQ, a prevenção das queimaduras bem como as complicações decorrentes das lesões teciduais e sistêmicas é ainda a estratégia de maior efetividade. Uma elevada incidência de queimaduras em crianças demanda programas de prevenção, especialmente em escolas e grupos comunitários de pais, identificados como corresponsáveis desses acidentes (CARNEIRO JG, et al., 2021).

Há formas inovadoras com boa aceitação e com resultados positivos no tratamento da dor e suas consequências na pediatria, como a realidade virtual. Família e equipe de enfermagem reconhecem os benefícios para distrair e tranquilizar a criança durante a realização do curativo (SCAPIN S, et al., 2020), esta tecnologia já se revela como opção não farmacológica para o alívio da dor para crianças queimadas (MARQUES LM, et al., 2021).

\section{CONCLUSÃO}

Os resultados do presente estudo indicam maiores incidências de queimaduras em crianças entre 1-2 anos, fato que revela como a curiosidade das crianças, a habilidade motora e a falta de medidas protetivas dos pais são fatores que contribuem para os acidentes. No decorrer do crescimento das crianças e adolescentes alguns agentes causais mudaram e outros se mantiveram, o que justifica a necessidade de abordagens preventivas que contemplem e valorizem as especificidades dos diferentes momentos infância e adolescência. As unidades básicas de saúde, escolas e poder público, de forma intersetorial, tem papel na divulgação de informações que conscientizem os responsáveis e as próprias crianças e adolescentes para a prevenção de queimaduras. Os profissionais da saúde, enquanto educadores, devem trabalhar nas consultas de puericultura, informações sobre queimaduras e prevenção, direcionando os pais para os riscos existentes em cada etapa de desenvolvimento da criança. 


\section{REFERÊNCIAS}

1. ANDRETTA IB, et al. Perfil epidemiológico das crianças internadas por queimaduras em hospital do sul do Brasil, de 1998 a 2008. Rev. Bras Queimaduras, 2013; 12 (1): 22-29.

2. ARRUDA FCF. Comparação de escores de gravidade para previsão de mortalidade e tempo de internação em unidade de queimados. Rev. Bras Queimaduras, 2017; 16(3): 142-149.

3. BARCELLOS LG, et al. Características e evolução de pacientes queimados admitidos em unidade de terapia intensiva pediátrica. Rev. Bras. Ter. Intensiva, 2018; 30(3): 333-337.

4. BRITO AR, et al. Acidentes com álcool em tempos de pandemia de covid-19. Rev. Ped. SOPERJ, 2021; 21(1): $01-06$.

5. CARNEIRO JG, et al. Perfil epidemiológico de vítimas de queimaduras internadas no Hospital de Emergência da Região Agreste de Alagoas. Revista de Saúde Coletiva da UEFS; 2021; 11(1): 5693.

6. CRUZ BEZERRA AF, et al. Mortalidade devido a queimaduras em crianças entre zero e quatro anos no Brasil. Brazilian Journal of Development, 2020; 6(7): 43012-43023.

7. FILHO RF, et al. Superfície corporal queimada vs. tempo de internação. Análise dos últimos 15 anos. Rev. Bras. Queimaduras, 2014; 13(1): 18-20.

8. FRANCISCONI MHG, et al. Perfil epidemiológico das crianças com queimaduras hospitalizadas em um Centro de Tratamento de Queimados. Rev. Bras. Queimaduras, 2016; 15(3): 137-141.

9. HERNANDEZ CMC, et al. Características de crianças hospitalizadas por queimaduras em um hospital em Manzanillo, Cuba. Rev. Bras. Queimaduras, 2018; (16)3: 169-173.

10. JUNIOR EML, et al. A influência dos aspectos socioeconômicos na ocorrência das queimaduras. Rev. Bras. Queimaduras, 2014; 13(1): 21-25.

11. JUNIOR EML, et al. Uso da pele de tilápia (Oreochromis niloticus), como curativo biológico oclusivo, no tratamento de queimaduras. Rev. Bras. Queimaduras, 2017; 16(1): 10-17.

12. LAITANO FF, et al. Contratura cicatricial severa em membros superiores após queimadura por escaldamento - relato de caso. Scientia Medica, 2013; 23(3): 180-183.

13. LEÃO CEG, et al. Epidemiologia das queimaduras no estado de Minas Gerais. Rev. Bras. Cir. Plást., $2011 ; 26$ (4): 573-577.

14. LEITE VHO, et al. Análise dos acidentes por queimadura com álcool líquido em Unidade de Tratamento de Queimados em Sergipe. Rev, Bras. Queimaduras, Sergipe, 2017; 15 (4): 235-239

15. LIMA JS, et al. Fatores de risco para infecção em crianças e adolescentes com queimaduras: estudo de coorte. Rev. Bras. Cir. Plást. 2016; 31(4): 545-553.

16. LIRA, PRB, et al. Perfil epidemiológico dos pacientes queimados atendidos em hospital de referência, PE, Brasil. Rev. Conbracis, 2017; 2(1): 1-9.

17. LUCENA, EVB; FIGUEIREDO, TP. Queimadura na infância: uma abordagem acerca das implicações para a saúde e qualidade de vida. Rev. Temas em saúde, 2017; 17(1): 245-261.

18. MARCHETI MA, et al. Accidents in childhood in times of COVID-19 pandemic. Rev. Soc. Bras. Enferm. Ped, 2020; 20: 16-25.

19. MARQUES LM, et al. Realidade virtual no controle da dor em pacientes pediátricos queimados. Revista Eletrônica Acervo Saúde, 2021; 13(3): 6696.

20. MESCHIAL WC, et al. Internação e mortalidade hospitalar de vítimas de queimaduras no Brasil. Revista Enfermagem Atual In Derme, 2020; 93(31): 1-8.

21. MINISTÉRIO DA SAÚDE. Datasus. Morbidade hospitalar do SUS por causas externas. 2018. Disponível em: http://tabnet.datasus.gov.br/cgi/tabcgi.exe?sih/cnv/fiuf.def. Acessado em: 11 de novembro de 2018.

22. SANCHES PHS, et al. Perfil epidemiológico de crianças atendidas em uma Unidade de Tratamento de Queimados no interior de São Paulo. Rev. Bras. Queimaduras, 2017; 15(4): 246-250.

23. SANTANA ME, et al. Perfil clínico e epidemiológico de crianças com queimaduras em um hospital de referência. Rev. Enferm. UFPI, 2018; 7(2): 23-27.

24. SANTOS CA, SANTOS AA. Assistência de enfermagem no atendimento pré-hospitalar ao paciente queimado: uma revisão da literatura. Rev. Bras. Queimaduras, 2017; 16(1): 28-33.

25. SCAPIN S, et al. Realidade virtual como tratamento complementar no alívio da dor em crianças queimadas. Texto \& Contexto-Enfermagem, 2020; 29: 1-15.

26. SILVA IKM, et al. Análise de pacientes de 0 a 12 anos atendidos no Pronto Socorro para Queimaduras de Goiânia em 2011 e 2012. Rev. Bras. Queimaduras, 2015; 14(1): 14-17.

27. SILVA RLM, et al. Características epidemiológicas das crianças vítimas de queimaduras atendidas no Hospital de Urgências de Sergipe. Rev. Bras. Queimaduras, 2016; 15(3): 158-163.

28. TAKINO MA, et al. Perfil epidemiológico de crianças e adolescentes vítimas de queimaduras admitidos em centro de tratamento de queimados. Rev. Bras. Queimaduras, 2016; 15(2): 74-79.

29. TONDINELI TH, et al. Queimaduras elétricas por alta voltagem: cinco anos de análise epidemiológica e tratamento cirúrgico atualizado. Rev. Bras. Cir. Plást, 2016; 31(3): 380-384. 